\title{
(C) OPEN ACCESS \\ Trichomonas vaginalis and HIV infection acquisition: a systematic review and meta-analysis
}

\author{
Simon Chengo Masha, ${ }^{1,2,3}$ Piet Cools, ${ }^{2}$ Eduard J Sanders, ${ }^{1,4}$ Mario Vaneechoutte, ${ }^{2}$ \\ Tania Crucitti $^{5}$
}

\begin{abstract}
- Additional material is published online only. To view please visit the journal online (http://dx.doi.org/10.1136/ sextrans-2018-053713)
\end{abstract}

${ }^{1}$ Centre for Geographic Medicine Research - Coast, Kenya Medical Research Institute, Kilifi, Kenya ${ }^{2}$ Laboratory for Bacteriology Research, Faculty of Medicine and Health Sciences, Ghent University, Ghent, Belgium ${ }^{3}$ Department of Biological Sciences, Pwani University, Kilifi, Kenya

${ }^{4}$ Nuffield Department of Clinical Medicine, University of Oxford, Oxford, UK

${ }^{5}$ HIV/STI Reference Laboratory, Department of Clinical Sciences, Institute of Tropical Medicine, Antwerpen, Belgium

\section{Correspondence to} Simon Chengo Masha, Centre for Geographic Medicine Research - Coast, Kenya Medical Research Institute, Kilifi, Kenya; schengo@gmail.com

SCM and PC shared first authorship.

Received 7 June 2018 Revised 20 August 2018 Accepted 18 September 2018 Published Online First 19 October 2018

\section{ABSTRACT \\ Objectives Trichomoniasis is the most prevalent curable STI globally, with the highest incidence and prevalence in sub-Saharan Africa (SSA). STIs have largely been associated with an increase in HIV acquisition. Our objective was to assess the existing literature available in English regarding the association of Trichomoniasis and HIV-1 acquisition.}

Methods The review protocol was registered at the International Prospective Register of Systematic Reviews (PROSPERO) under number CRD42018082702. We searched MEDLINE, Embase and Scopus databases to collect articles measuring the association of Trichomonas vaginalis infection and HIV acquisition and performed a meta-analysis and qualitative synthesis of the literature. Results We identified 1806 unduplicated citations, of which 18 papers and 1 conference abstract were eligible for inclusion in the review after applying our inclusion and exclusion criteria. All the studies included in the systematic review had been carried out in SSA. The articles reported various measures of effects, namely: $\mathrm{HRs}$, rate ratios, risk ratios and ORs. In a meta-analysis restricted to 11 studies reporting $H R$, individuals infected with $T$. vaginalis were 1.5 times more likely to acquire HIV compared with individuals not infected with $T$. vaginalis $(95 \% \mathrm{Cl} 1.3$ to $1.7 ; \mathrm{p}<0.001)$.

Conclusions $T$. vaginalis is an important factor in HIV acquisition especially in SSA where the prevalence of both $T$. vaginalis and HIV-1 are high. This systematic review and meta-analysis confirms the evidence that infection with $T$. vaginalis augments HIV acquisition with $50 \%$. Diagnosis and treatment of $T$. vaginalis infection in both high-risk and low-risk individuals may be a potential tool to reduce new HIV infections.

Trial registration number CRD42018082702

\section{INTRODUCTION}

The protozoan Trichomonas vaginalis is responsible for trichomoniasis, the most common curable STI. ${ }^{1}$ T. vaginalis infects both men and women, although this unicellular parasite is more prevalent in women than men. Among people aged 15-49 years, it was estimated that in 2012 the global prevalence of $T$. vaginalis infection among women was $5.0 \%$ versus $0.6 \%$ among men. These figures correspond to an estimated incidence of 143 million newly infected individuals. ${ }^{1}$ Similar to HIV, T. vaginalis is more prevalent in sub-Saharan Africa (sSA), as the cumulative prevalence was estimated to be $11.5 \%$ among women aged between 15 years and 49 years in sSA in $2012 .^{1}$
According to The Joint United Nations Programme on HIV and AIDS, ${ }^{2}$ about 5000 new HIV infections occurred daily in 2016, with a huge proportion, that is, $64 \%$, of new infections occurring in sSA. Already two decades ago, reports suggested that individuals infected with $T$. vaginalis were at an increased risk of HIV acquisition. ${ }^{3}$ Some studies have also indicated that T. vaginalis and HIV coinfection increases genital shedding of $\mathrm{HIV}^{4}{ }^{4}$ but opinions differ, especially in the context of antiretroviral therapy. ${ }^{5}$ In summary, there is growing evidence that there is a need for special treatment considerations for $T$. vaginalis among women with HIV coinfection. ${ }^{6}$

Better understanding of the association $T$. vaginalis and HIV may provide insights on HIV infections that may be attributed to $T$. vaginalis. Moreover, we may learn how control of T. vaginalis may impact HIV transmission as a potentially cost-effective strategy for reducing HIV transmission, particularly in regions where $T$. vaginalis is common. ${ }^{7}$ The objective of this systematic review and meta-analysis was to clarify the extent to which T. vaginalis infection is associated with HIV infection and acquisition.

\section{METHODS}

\section{Protocol and registration}

We followed the Preferred Reporting Items for Systematic Reviews and Meta-Analyses (PRISMA) guidelines $^{8}$ for carrying out and reporting this systematic review and meta-analysis, using an a priori defined protocol.

\section{Eligibility criteria}

We included studies that reported both T. vaginalis infection status and HIV status. We only included studies written in English and that documented $\mathrm{OR}$, or risk ratio (RiR), or HRs, or provided the absolute numbers for these parameters to be calculated. There were no restrictions in terms of study design or geographical area where the studies were conducted. We included papers that were published up to 11 March 2018.

Reviews, narrative-only conference abstracts, comments, guidelines, case reports or case series, unpublished articles, multiple reports of the same data and in vitro and animal studies were not considered for inclusion. Infection with $T$. vaginalis was defined as the presence of the pathogen as assessed by culture, wet mount or molecular methods, while HIV status was defined by the presence of HIV antibodies, HIV proteins, HIV RNA or DNA. 


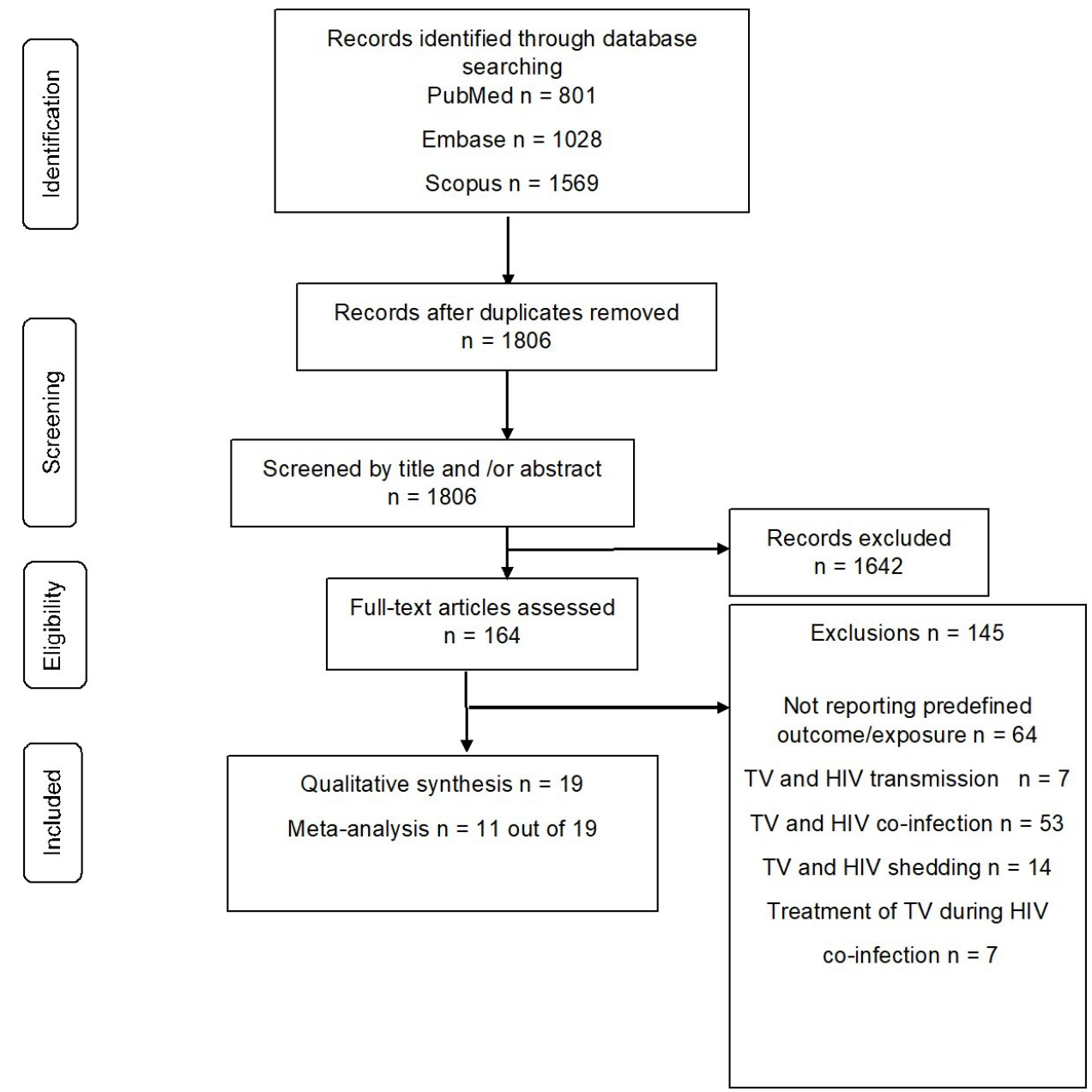

Figure 1 Identification of studies included in the systematic review. HIV, human immunodefeciency virus; TV, Trichomonas vaginalis

\section{Search strategy}

We searched Embased, MEDLINE (through PubMed) and Scopus. We used the combination of all of the following search terms (Trichomonas vaginalis, T. vaginalis and trichomoniasis) with all of the following search terms (AIDS, acquired immunodeficiency syndrome, HIV, HIV, HIV-1 and HIV-2). The bibliographic references of the studies that were considered eligible were also hand searched.

\section{Study selection}

Similar to the systematic review by Cools et al, ${ }^{9}$ studies were selected in a two-stage process (figure 1). First, all bibliographic references were screened to identify studies for full-text evaluation by one reviewer (SCM), on the basis of only the information present in the title and abstract. Subsequently, the full texts of those articles not excluded in the screening process were assessed for eligibility, using the aforementioned criteria, by two authors independently (SCM and PC). Reasons for exclusion were recorded and categorised, and disagreements were resolved by consensus.

Using all search terms, searches were conducted for relevant papers in the preidentified databases (Embase, PubMed and Scopus), and from this, we identified 3398 papers. Once the search was completed, all of the retrieved articles were imported into EndNote X7.8 for removal of duplicates.

\section{Data collection process}

A predefined data extraction form was pilot-tested and used for data extraction. From the 19 studies included in the systematic review and meta-analysis, the following information was collected: authors, year of publication, journal, country where the study had been carried out, study design, sample collection period, study population, mean/median age of the participants, total number of study participants, total number of samples included in the analysis, T. vaginalis detection method, assessment of HIV status, relevant measures of effects or absolute numbers and statistics. This procedure was performed independently by two authors (SCM and PC), and disagreements were resolved by discussion between both authors.

\section{Risk of bias in individual studies}

To assess the quality and risk of bias of the included studies, we developed a quality appraisal tool (online supplementary table 1) based on the Newcastle-Ottawa Scale. ${ }^{10}$ We adapted this tool to the content of our meta-analysis. Stars were assigned by SCM for three broad criteria (representativeness of the study groups, comparability of the study groups and quality of the outcome assessment) in order to provide a measure of their quality.

Studies that scored none or one, two or three stars for internal validity of representativeness were classified as having respectively a high, medium or low risk of bias for this criterion. 
Studies assigned none, one or two stars for comparability or outcome were considered to have respectively a high, medium or low risk of bias for comparability and outcome. ${ }^{9}$

\section{Data analysis}

The meta-analyses was performed using STATA V.13.1. We reported the results of the meta-analyses obtained after pooling individual study estimates with random effects model as HRs with 95\% CI. The degree of heterogeneity between the studies was assessed using the $\mathrm{I}^{2}$ index, with percentages $25 \%, 50 \%$ and $75 \%$ being indicative of low, moderate and high heterogeneity, respectively. ${ }^{11}$

To assess publication bias, a funnel plot was created by plotting the natural logarithm of the HRs against the inverse of the SE. The symmetry of the funnel plots was visually inspected and statistically checked using Egger's regression test. ${ }^{12}$

\section{RESULTS}

\section{Manuscript and study selection}

A total of 1806 unduplicated citations were identified; we selected 164 abstracts for full-text evaluation (figure 1). There were 19 studies that met our inclusion criteria to be included in our systematic review.

\section{Description of selected studies}

All studies were conducted in sSA, namely Burkina Faso $(n=1)$, Democratic Republic of Congo $(n=2)$, Ivory Coast $(n=1)$, Kenya $(n=5)$, Malawi $(n=1)$, South Africa $(n=4)$, Uganda $(n=4)$, Zambia $(n=1)$ and Zimbabwe $(n=3)$. Some of the studies were conducted in more than one country. Of the included 19 studies, 17 were cohorts and 2 were nested case-control studies. Generally, all these studies involved a baseline $T$. vaginalis test and additional testing performed at varying intervals depending on the study, with treatment for participants testing positive for T. vaginalis.

Thirteen of the studies found a positive association between $T$. vaginalis infection and HIV acquisition.

Eleven studies reported HRs of HIV acquisition in relation to T. vaginalis infection, three reported RRs (ie, comparison of incidence rates) of HIV acquisition in relation to T. vaginalis, two studies reported RiRs of HIV acquisition in relation to T. vaginalis and three reported ORs of HIV acquisition in relation to $T$. vaginalis (table 1 ).

Most studies (63\%) had been carried out among high-risk individuals, that is, female sex workers, and serodiscordant couples. The rest of the studies (37\%) had been carried out among low-risk populations, including women attending prenatal or postnatal clinics and family planning clinics and individuals from the general population. Only three studies had male participants. Direct microscopy was the most common method used for detection of T. vaginalis (73\%) in the studies. Detection of HIV antibodies was the most common method used to determine HIV status (84\%) in the studies (table 1).

Treatment of $T$. vaginalis in the studies included in the meta-analysis was mostly on the basis of metronidazole. However, not all participants infected with T. vaginalis received treatment. For instance, in the study by Van Der Pol et $a l^{13}$, participants diagnosed using microscopy received treatment, while those diagnosed using nucleic acid amplification test (NAAT) were not treated. Additionally, in the study by Kleinschmidt $e t a l^{14}$ syndromic treatment of STIs was offered.

\section{Risk of bias within studies}

Following the critical appraisals of the included studies (online supplementary figure 1). Fourteen studies were assessed as having a high risk of bias for representativeness (high-risk individuals, that is, female sex workers, and serodiscordant couples), three as having a medium risk of bias for representativeness (prenatal care clinic/postnatal care clinic women and family planning clinic attendees) and two studies as having a low risk of bias for representativeness (women from the general population). For the risk of bias for outcome assessment, it was high for 1 study, medium for 16 studies and low for 2 studies. Eight out of the 19 studies were assessed as having a medium risk of bias for comparability, while 11 studies had a low risk of bias for comparability. Individual study appraisals are captured in online supplementary table 2 .

Visual inspection of the funnel plot shows symmetrical distribution of studies, suggesting no publication bias (figure 2), which is supported by Egger's regression intercept $(0.6 ; 95 \% \mathrm{CI}-0.64$ to $1.82 ; \mathrm{p}=0.304)$.

\section{Association of $T$. vaginalis and HIV acquisition}

Eleven of the studies that were reporting HR provided data for the meta-analysis. The point estimate for the HR ranged from 0.71 to 4.79 (table 1). Bearing in mind that ORs and RRs measure only the number of events and take not into account when they occur, these two measures are appropriate for measuring dichotomous outcomes but less appropriate for analysing time-to-event outcomes, which is important when relating T. vaginalis infection and HIV acquisition. The meta-analysis indicated that individuals infected with $T$. vaginalis were 1.5 times more likely to acquire HIV as compared with individuals not infected with $T$. vaginalis (HR: 1.5 ; 95\% CI 1.3 to $1.7 ; \mathrm{p}<0.001$ ) (figure 3 ).

\section{DISCUSSION}

In this systematic review and meta-analysis, we assessed the extent to which $T$. vaginalis infection is associated with HIV acquisition. There has been a global decline in deaths from HIV/ AIDS-related causes, from a peak of 1.9 million in 2005 to 1.0 million in $2016 .^{2}$ Despite this, AIDS-related illnesses remain the leading cause of death among women of reproductive age (15-49 years) globally ${ }^{2}$ and the second leading cause of death for young women aged 15-24 years in sSA. ${ }^{2}$ The prevalence of T. vaginalis was estimated to be approximately $11 \%$ in 2012 among women in sSA. ${ }^{1}$

Studies included in our meta-analysis had no heterogeneity and thus appropriate to be combined in a meta-analysis where we demonstrate that individuals infected with $T$. vaginalis are at a higher risk of HIV acquisition. T. vaginalis augments the likelihood of HIV acquisition by 50\% (HR 1.5 ; 95\% CI 1.3 to 1.7 ); the amount of HIV acquisition attributable to this pathogen (attributable risk) and its contribution to the HIV pandemic may be substantial due to the high prevalence of $T$. vaginalis infection, especially in sSA. Our results are in agreement with a previous systematic review where they also show infection with T. vaginalis increased risk of HIV acquisition. ${ }^{15}$

In a study where they included both high-risk individuals and low-risk individuals, the adjusted HR for HIV acquisition for women with T. vaginalis were higher in the low-risk individuals, suggesting that for women with less risk from sexual networks, infection with $T$. vaginalis infection is a very important risk factor for HIV acquisition. ${ }^{13}$

Most studies included in this systematic review used direct microscopy for detection of $T$. vaginalis, a diagnostic technique that has been shown to have a low sensitivity. ${ }^{13}$ In a study where they included both high-risk individuals and low-risk individuals, the adjusted HR for HIV acquisition for women with T. 


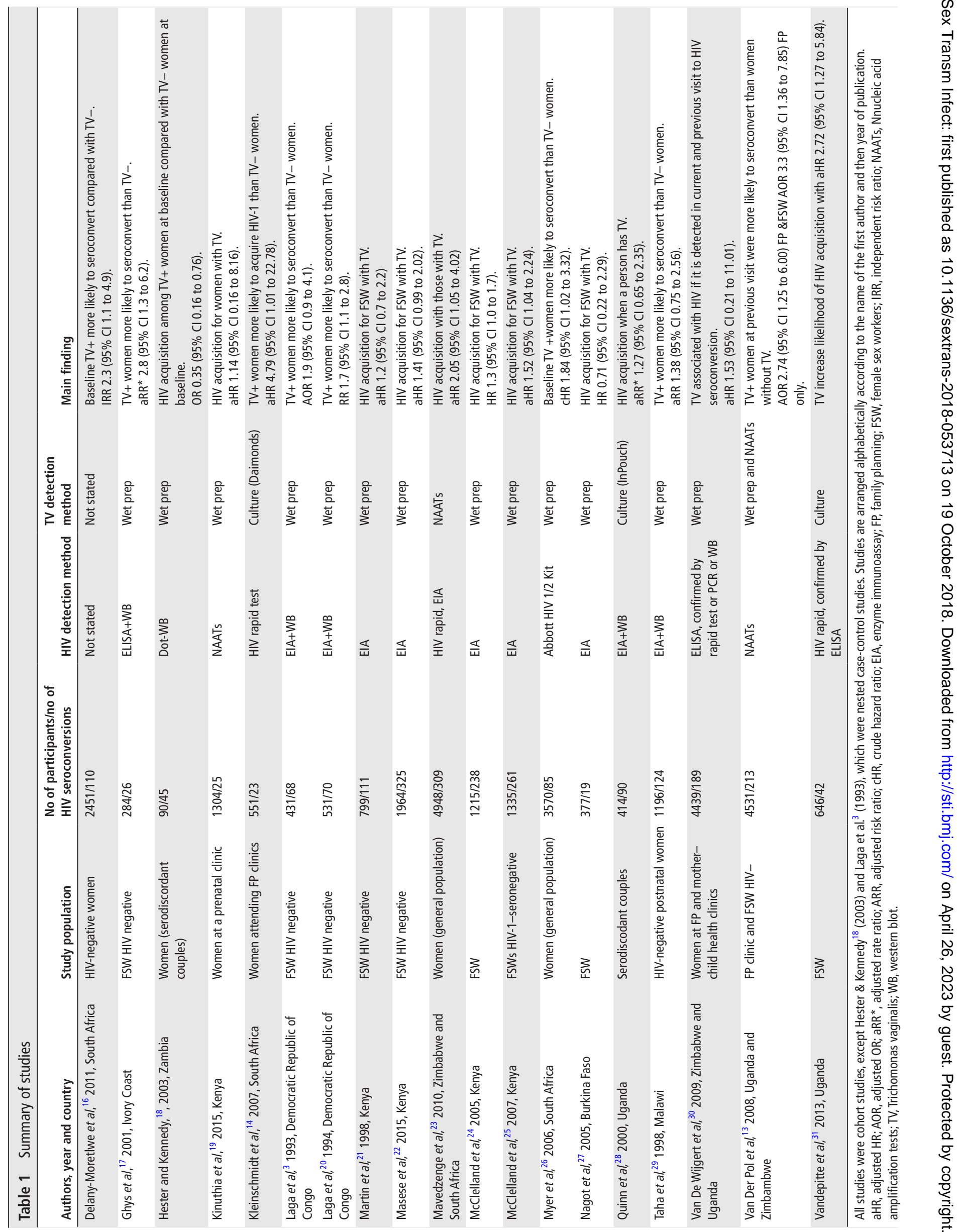




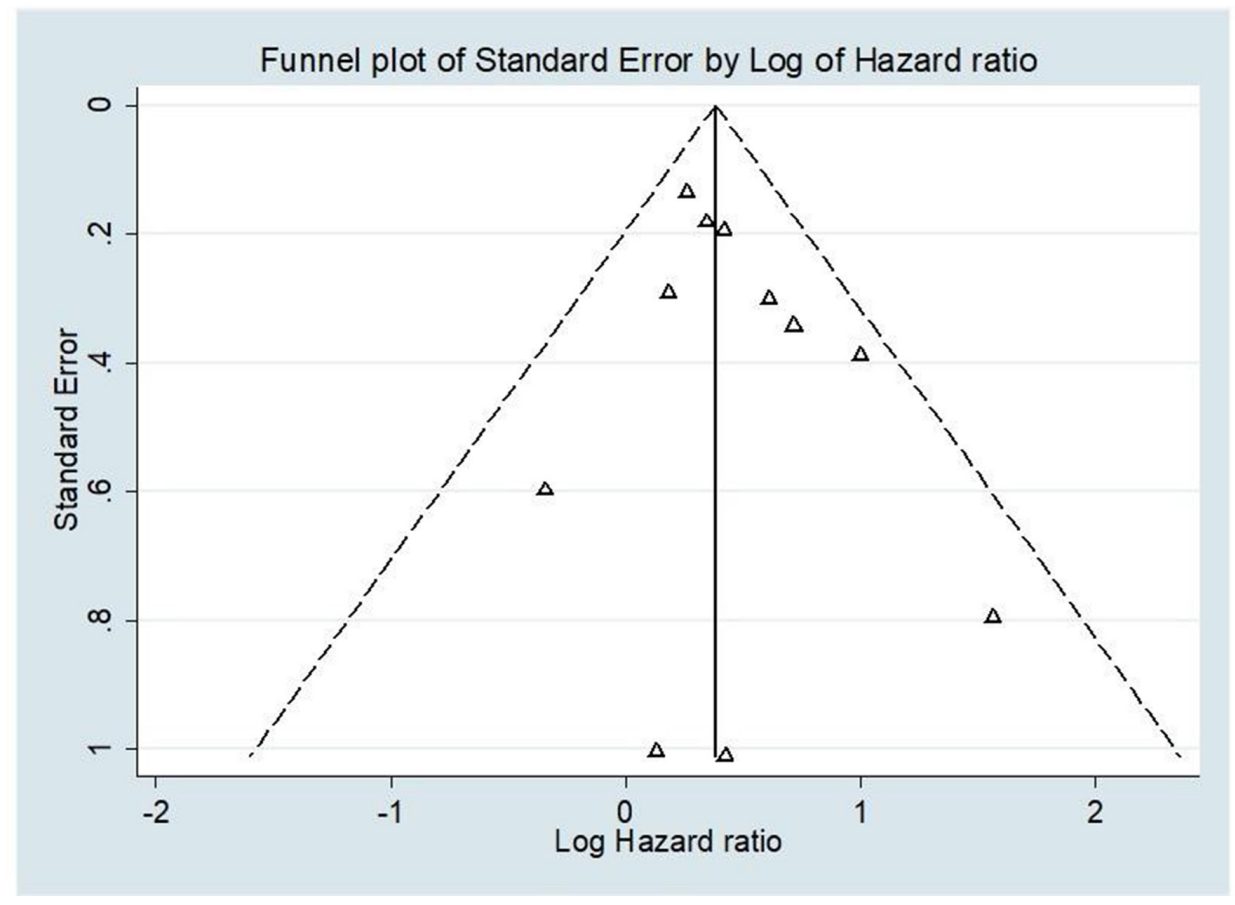

Figure 2 Funnel plot to assess publication bias among studies evaluating the association between Trichomonas vaginalis infection and HIV infection acquisition. The triangles represent the estimates of the 11 included studies of the association between $T$. vaginalis infection and HIV infection acquisition. The log of the HR is plotted on the horizontal axis, against the inverse of the SE of the HR on the vertical axis. The vertical line in the funnel plot indicates the fixed-effects summary estimate and the sloping lines indicate the expected 95\% Cls for a given SE.

vaginalis were higher in the low-risk individuals, suggesting that for women with less risk from sexual networks, infection with T. vaginalis infection is a very important risk factor for HIV ${ }^{13}$

Several $T$. vaginalis pathogenic mechanisms of $T$. vaginalis may help to explain why individuals infected with $T$. vaginalis may be at a higher risk of HIV acquisition. First, T. vaginalis may damage the epithelial membrane ${ }^{\mathrm{w} 2}$, which acts as a barrier to HIV through several pathogenic mechanisms; its cell-to-cell adhesion $^{\mathrm{w} 3}$, haemolysis ${ }^{\mathrm{w} 4}$ and/or excretion of soluble factors ${ }^{\mathrm{w} 5}$. Second, T. vaginalis has been shown to elicit an inflammatory

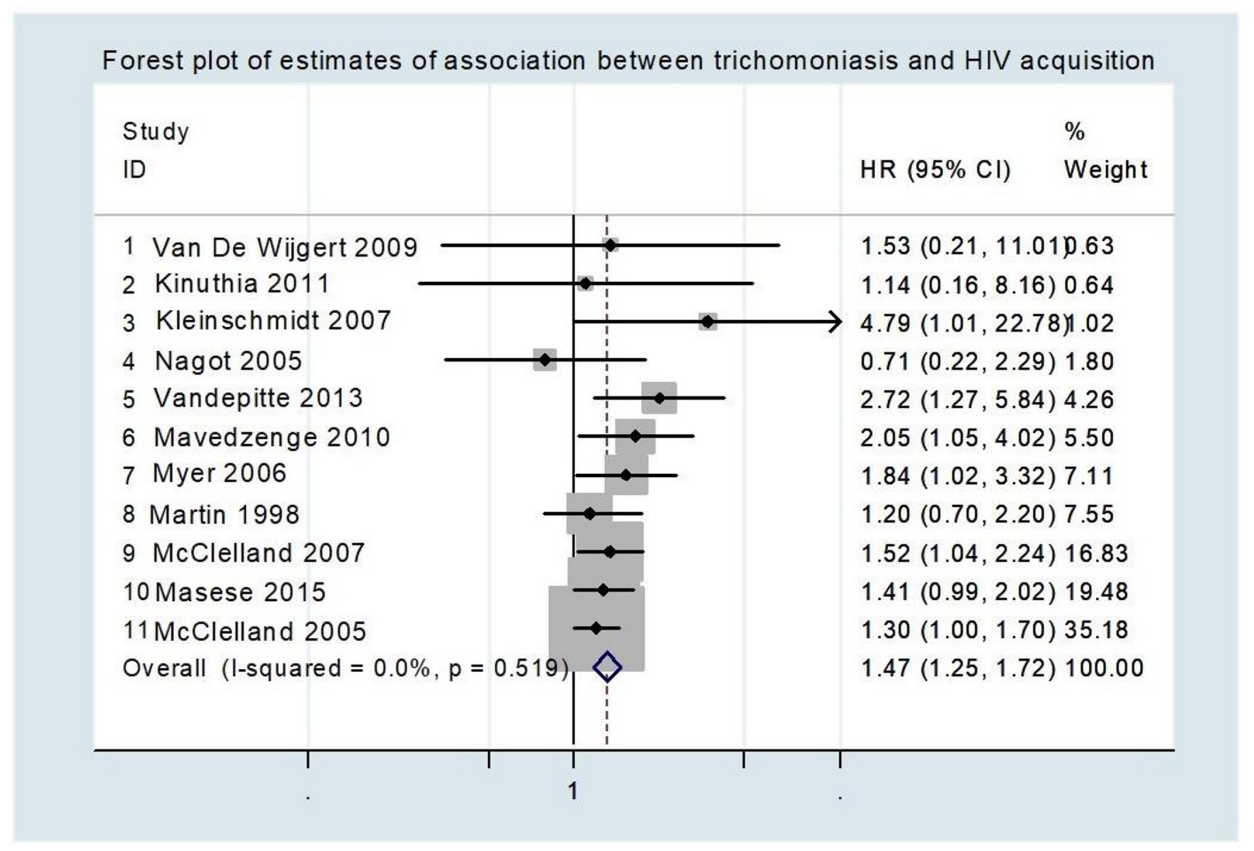

Figure 3 Forest plot of estimates of association between trichomoniasis and HIV acquisition. Studies are plotted to start with the one with the least weight. Each study is represented by a black circle and a horizontal line, which correspond to the $\mathrm{HR}$ and $95 \% \mathrm{Cl}$, respectively. The area of the grey box around each study reflects the weight of the study (determined by random effects analysis) in the meta-analysis. The vertical line in the middle corresponds to an HR of 1.0. The diamond represents the overall HR with the $95 \% \mathrm{Cl}$ given by its width. The arrow indicates the study with the highest confidence interval. There was low heterogeneity of studies included in the meta-analysis as shown by the $\mathrm{I}^{2}=0.0 \%, \mathrm{p}=0.510$. 
response among infected individuals this may lead to an increase in the appearance of HIV target cells ${ }^{\mathrm{w} 6, \mathrm{w} 7}$. Third, infection with $T$. vaginalis has been associated with bacterial vaginosis ${ }^{\mathrm{w} 8}$ which, in turn, can increase the risk of HIV acquisition ${ }^{\text {w9 }}$. All these consequences may facilitate HIV in T. vaginalis-infected individuals.

In cases of HIV and T. vaginalis coinfection, T. vaginalis has been associated with increased genital shedding of $\mathrm{HIV}^{\mathrm{w} 10}$. A previous study showed high prevalence of $T$. vaginalis among HIV serodiscordant African couples ${ }^{\mathrm{w} 11}$. T. vaginalis may be an important factor for HIV transmission in HIV discordant couples. Moreover, if both individuals are infected with T. vaginalis as there is potential for synergistic effect, that is, increased genital shedding of HIV, combined with pathogenic mechanisms of $T$. vaginalis, which potentially make individuals infected with T. vaginalis be at a higher risk of HIV acquisition. Theoretically, this would increase the likelihood of HIV acquisition than when only one the individuals is infected with $T$. vaginalis.

Regarding the limitations of this review, first, most studies included in the review were conducted among high-risk individuals, that is, female sex workers, and serodiscordant couples. Thus, our result may not reflect the situation in the general population. Second, only a couple of studies included in the review had male participants, but all the studies in our meta-analysis were based on women; our results are biased towards high-risk females. Third, most of the studies in the review used direct microscopy to detect $T$. vaginalis infection. Direct microscopy is characterised by low sensitivity, and samples detected through this technique usually have high parasitaemia. Although the few studies that used culture or NAATs for T. vaginalis detection reported positive association of $T$. vaginalis infection and increased likelihood of HIV acquisition, they also signal the need for further longitudinal research on this topic, evaluating if $T$. vaginalis detection techniques have an impact of this association Fourth, we did not assess the risk of bias that might be due to missing data or loss to follow-up. Finally, all studies included in the review were done in sSA, and thus our results may only apply to the sSA population.

\section{CONCLUSION}

T. vaginalis may be used as a biological marker for enhanced risk for HIV acquisition for both high-risk and moderate-risk women. T. vaginalis may be an important factor in HIV acquisition especially in sSA where the prevalence of both T. vaginalis and HIV is high, and this systematic review and meta-analysis provides consolidated evidence that infection with $T$. vaginalis augments HIV acquisition. Diagnosis and treatment of T. vaginalis in both individuals at high risk and low risk for HIV may perhaps be a potential tool to reduce new HIV infections.

Additional references can be found in the online supplementary file 2 .

\section{Key messages}

- Trichomoniasis augments the likelihood of HIV acquisition by $50 \%$.

- Trichomoniasis may be an important factor in HIV acquisition especially in sub-Saharan Africa where the prevalence of both are high.

- Screening and treatment of trichomoniasis may be a potential means of curbing new HIV infections among women with high-risk or moderate-risk behaviour.

\section{Handling editor Jackie A Cassell}

Acknowledgements This manuscript was submitted for publication with permission from the Director of the Kenya Medical Research Institute (KEMRI).

Contributors SCM, PC and TC designed the study. SCM and PC searched for literature, reviewed titles and abstracts for inclusion in the review, performed data extraction and conducted the analysis. SCM wrote the first draft of the manuscript. All authors contributed to data interpretation, reviewed successive drafts and approved the final version of the manuscript.

Funding This research has been supported by a PhD Scholarship for SCM from the Belgian Development Cooperation through VLIR-UOS. The funder had no role in the in the design of the study and collection, analysis, and interpretation of data and in writing the manuscript. The views expressed here are those of the authors and do not necessarily represent the views of the Belgian Development Cooperation.

\section{Competing interests None declared.}

Provenance and peer review Not commissioned; externally peer reviewed.

Open access This is an open access article distributed in accordance with the Creative Commons Attribution Non Commercial (CC BY-NC 4.0) license, which permits others to distribute, remix, adapt, build upon this work non-commercially, and license their derivative works on different terms, provided the original work is properly cited, appropriate credit is given, any changes made indicated, and the use is non-commercial. See: http://creativecommons.org/licenses/by-nc/4.0

\section{REFERENCES}

1 Newman L, Rowley J, Vander Hoorn S, et al. Global estimates of the prevalence and incidence of four curable sexually transmitted infections in 2012 based on systematic review and global reporting. PLoS One 2015;10:e0143304.

2 UNAIDS, 2017. http://www.unaids.org/en/resources/documents/2017/2017_data_ book (accessed 06 Jun 2018).

3 Laga M, Manoka A, Kivuvu M, et al. Non-ulcerative sexually transmitted diseases as risk factors for HIV-1 transmission in women: results from a cohort study. AIDS 1993:7:95-102.

4 Kissinger $\mathrm{P}$, Amedee A, Clark RA, et al. Trichomonas vaginalis treatment reduces vaginal HIV-1 shedding. Sex Transm Dis 2009:36:11-16.

5 Masese LN, Graham SM, Gitau R, et al. A prospective study of vaginal trichomoniasis and HIV-1 shedding in women on antiretroviral therapy. BMC Infect Dis 2011;11:307.

6 Howe K, Kissinger PJ. Single-dose compared with multidose metronidazole for the treatment of trichomoniasis in women: a meta-analysis. Sex Transm Dis 2017:44:30-5

7 Price MA, Stewart SR, Miller WC, et al. The cost-effectiveness of treating male trichomoniasis to avert HIV transmission in men seeking sexually transmitted disease care in Malawi. J Acquir Immune Defic Syndr 2006;43:202-9.

8 Moher D, Liberati A, Tetzlaff J, et al. Preferred reporting items for systematic reviews and meta-analyses: the PRISMA statement. PLoS Med 2009;6:e1000097.

9 Cools P, van de Wijgert J, Jespers $\mathrm{V}$, et al. Role of HIV exposure and infection in relation to neonatal GBS disease and rectovaginal GBS carriage: a systematic review and meta-analysis. Sci Rep 2017;7:13820.

10 Zeng X, Zhang Y, Kwong JS, et al. The methodological quality assessment tools for preclinical and clinical studies, systematic review and meta-analysis, and clinical practice guideline: a systematic review. J Evid Based Med 2015;8:2-10.

11 Higgins JP, Thompson SG, Deeks JJ, et al. Measuring inconsistency in meta-analyses. BMJ 2003;327:557-60.

12 Egger M, Davey Smith G, Schneider M, et al. Bias in meta-analysis detected by a simple, graphical test. BMJ 1997;315:629-34.

13 Van Der Pol B, Kwok C, Pierre-Louis B, et al. Trichomonas vaginalis infection and human immunodeficiency virus acquisition in African women. $J$ Infect Dis 2008;197:548-54.

14 Kleinschmidt I, Rees H, Delany S, et al. Injectable progestin contraceptive use and risk of HIV infection in a South African family planning cohort. Contraception 2007:75:461-7.

15 Kissinger P, Adamski A. Trichomoniasis and HIV interactions: a review. Sex Transm Infect 2013;89:426-33.

16 Delany-Moretlwe S, Nanoo A, Nagpal A, et al. P1-S5.14 Risk factors associated with HIV acquisition: a comparative analysis of older and younger women who participated in the MDP301 trial in Johannesburg. Sex Transm Infect 2011;87(Suppl 1):A179-A180.

17 Ghys PD, Diallo MO, Ettiègne-Traoré $\mathrm{V}$, et al. Effect of interventions to control sexually transmitted disease on the incidence of HIV infection in female sex workers. AIDS 2001;15:1421-31.

18 Hester RA, Kennedy SB. Candida infection as a risk factor for HIV transmission. J Womens Health 2003;12:487-94.

19 Kinuthia J, Drake AL, Matemo D, et al. HIV acquisition during pregnancy and postpartum is associated with genital infections and partnership characteristics. AIDS 2015;29:2025-33. 
20 Laga M, Alary M, Nzila N, et al. Condom promotion, sexually transmitted diseases treatment, and declining incidence of HIV-1 infection in female Zairian sex workers. Lancet 1994;344:246-8.

21 Martin HL, Nyange PM, Richardson BA, et al. Hormonal contraception, sexually transmitted diseases, and risk of heterosexual transmission of human immunodeficiency virus type 1. J Infect Dis 1998;178:1053-9.

22 Masese L, Baeten JM, Richardson BA, et al. Changes in the contribution of genital tract infections to HIV acquisition among Kenyan high-risk women from 1993 to 2012. AIDS 2015;29:1077-85.

23 Mavedzenge SN, Pol BV, Cheng $\mathrm{H}$, et al. Epidemiological synergy of Trichomonas vaginalis and HIV in Zimbabwean and South African women. Sex Transm Dis 2010;37:460-6.

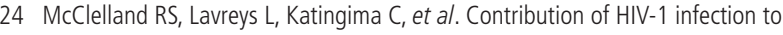
acquisition of sexually transmitted disease: a 10-year prospective study. J Infect Dis 2005; 191:333-8.

25 McClelland RS, Sangaré L, Hassan WM, et al. Infection with Trichomonas vaginalis increases the risk of HIV-1 acquisition. J Infect Dis 2007;195:698-702.
26 Myer L, Denny L, de Souza M, et al. Distinguishing the temporal association between women's intravaginal practices and risk of human immunodeficiency virus infection: a prospective study of South African women. Am J Epidemiol 2006;163:552-60.

27 Nagot N, Ouedraogo A, Ouangre A, et al. Is sexually transmitted infection management among sex workers still able to mitigate the spread of HIV infection in West Africa? J Acquir Immune Defic Syndr 2005;39:454-8.

28 Quinn TC, Wawer MJ, Sewankambo N, et al. Viral load and heterosexual transmission of human immunodeficiency virus type 1. Rakai Project Study Group. N Engl J Med 2000;342:921-9.

29 Taha TE, Hoover DR, Dallabetta GA, et al. Bacterial vaginosis and disturbances of vaginal flora: association with increased acquisition of HIV. AIDS 1998;12:1699-706.

30 van de Wijgert JH, Morrison CS, Brown J, et al. Disentangling contributions of reproductive tract infections to HIV acquisition in African Women. Sex Transm Dis 2009;36:357-64.

31 Vandepitte J, Weiss HA, Bukenya J, et al. Alcohol use, mycoplasma genitalium, and other STIs associated with HIV incidence among women at high risk in Kampala, Uganda. J Acquir Immune Defic Syndr 2013;62:119-26. 